\title{
Review of: "Enabling countries to apply behavioural science in using global survey data to inform their Covid-19 policies"
}

Laisa Liane Paineiras-Domingos

Potential competing interests: The author(s) declared that no potential competing interests exist.

Congratulations. This work is relevant because present originality and a big public health interest. The title could be revised to better highlight the proposal and objective of the study. Its aims are clear and the methodology adequate. Data analysis must to be more clear and the results are easy to understand with good illustrative tables. I think the most ideal description would be tables and not figures $1,2,3$. The authors used very relevant references, which guarantees a good basis in the theoretical construction of the proposal presented. 